\title{
Evaluation of the association between the TAS1R2 and TAS1R3 variants and food intake and nutritional status in children
}

\author{
Silvia V. Melo ${ }^{1,2}$, Grasiela Agnes ${ }^{1}$, Márcia R. Vitolo ${ }^{2}$, Vanessa S. Mattevi ${ }^{1,2}$, Paula D.B. Campagnolo ${ }^{3}$ and \\ Silvana Almeida ${ }^{1,2}$ \\ ${ }^{1}$ Laboratório de Biologia Molecular, Universidade Federal de Ciências da Saúde de Porto Alegre, Porto \\ Alegre, RS, Brazil. \\ ${ }^{2}$ Programa de Pós-Graduação em Ciências da Saúde, Universidade Federal de Ciências da Saúde de Porto \\ Alegre, Porto Alegre, RS, Brazil. \\ ${ }^{3}$ Centro de Ciências da Saúde, Universidade do Vale dos Sinos (UNISINOS), Porto Alegre, RS, Brazil.
}

\begin{abstract}
Taste perception plays a key role in determining individual food preferences and dietary habits and may influence nutritional status. This study aimed to investigate the association of TAS1R2 (Ile191Val - rs35874116) and TAS1R3 (-1266 C/T - rs35744813) variants with food intake and nutritional status in children followed from birth until 7.7 years old. The nutritional status and food intake data of 312 children were collected at three developmental stages (1, 3.9 and 7.7 years old). DNA was extracted from blood samples and the polymorphisms were analyzed by real-time polymerase chain reactions (qPCR) using hydrolysis probes as the detection method. Food intake and nutritional status were compared among individuals with different single nucleotide polymorphism (SNP) genotypes. At 3.9 years old, children homozygous ( $\mathrm{Val} / \mathrm{Val}$ ) for the TAS1R2 lle191 Val polymorphism ingested less sugar and sugar-dense foods than children who were *lle carriers. This finding demonstrated that a genetic variant of the T1R2 taste receptor is associated with the intake of different amounts of high sugar-content foods in childhood. This association may provide new perspectives for studying dietary patterns and nutritional status in childhood.
\end{abstract}

Keywords: food intake, polymorphism, TAS1R2 gene, TAS1R3 gene, taste receptors.

Received: July 27, 2016; Accepted: November 23, 2016.

\section{Introduction}

The prevalence of childhood obesity throughout the world has increased rapidly in recent decades (Oliveira and Fisberg, 2003; Allison et al., 2008; WHO, 2010). The perception of flavors (sweet, bitter, salty, sour and umami) can play a key role in determining the preference for foods and dietary habits, thereby influencing nutritional status (Garcia-Bailo et al., 2009). Individual food preferences are based on physiological and nutritional factors, as well as economic and socio-cultural aspects. However, the sensory properties of food have a critical effect on dietary choices, and flavor can be a major determinant of food selection (Leterne et al., 2008; Garcia-Bailo et al., 2009). The T2R family of taste receptors is associated with the perception of bitter taste while the T1R family is associated with the perception of sweet and umami tastes (Nelson et al., 2001; Zhao et al., 2003). The T1R family consists of the T1R1,

Send correspondence to Silvana Almeida. Departamento de Ciências Básicas da Saúde, Universidade Federal de Ciências da Saúde de Porto Alegre, Rua Sarmento Leite, 245 - Prédio 1 sala 309, 90050-170, Porto Alegre, RS, Brazil. E-mail: salmeida@ufcspa.edu.br
T1R2 and T1R3 receptors that are coupled to a heterodimeric G protein (Hoon et al., 1999; Zhao et al., 2003). The T1R receptors combine to form heterodimeric receptor complexes: T1R1-T1R3 is responsive to L-amino acids (umami taste perception) and T1R2-T1R3 is responsive to a variety of molecules, including natural sugars and artificial sweeteners (perception of sweetness) (Zhao et al., 2003). The receptor complex T1R2-T1R3 is encoded by the TAS1R2 and TAS1R3 genes, both of which are located on chromosome 1 (Liao and Schultz, 2003; Fushan et al., 2009). The $T A S 1 R 2$ gene is characterized by a high natural diversity and the Ile191 Val polymorphism (rs35874116) leads to the substitution of an isoleucine for a valine at position 191, which lies in a putative ligand-binding domain. Eny et al. (2010) reported an association between this polymorphism and sugar intake and differences in the response to dietary counseling. Fushan et al. (2009) described two polymorphisms in the TAS1R3 gene, $-1572 \mathrm{C} / \mathrm{T}$ (rs307355) and $-1266 C / T$ (rs35744813), that are in strong linkage disequilibrium and could be related to the perception of the taste of sucrose; these polymorphisms account for $16 \%$ of the variation in sensibility. 
Genetic variation in taste receptors may be associated with greater sensitivity to particular flavors and result in a higher intake of some foods considered to be palatable but which are highly energetic and may be associated with an increased risk of overweight. The aim of this study was to examine the association of the Ile191Val SNP in the $T A S 1 R 2$ gene and the $-1266 C / T$ SNP in the TAS1R3 gene with food intake and nutritional status at three developmental phases in children followed up until 7.7 years old.

\section{Subjects and Methods}

\section{Subjects}

The subject cohort was drawn from a randomized trial conducted in the first year of life to assess the impact of dietary counseling given to mothers during the first year of the infants' lives and dealt with food consumption, nutritional status and lipid profile (Vitolo et al., 2010). The initial sample consisted of 500 children born from October 2001 to July 2002. Mothers were recruited from the maternity ward of the only publicly funded city hospital in São Leopoldo, a city in the southern Brazilian state of Rio Grande do Sul. HIV-positive mothers and infants with congenital malformations were excluded from the study. All eligible mothers were informed by field workers about the overall aims of the study and all research procedures. The intervention group received dietary advice during home visits in the first year of life, based on the 'Ten Steps to Healthy Feeding', a Brazilian national health policy for primary care, supported by the World Health Organization (Brasil Ministério da Saúde and Organização Pan-Americana da Saúde, 2002). The dietary advice encouraged exclusive breastfeeding during the first six months, after which the mothers were encouraged to continue breastfeeding and gradually introduce foods. Both groups (control and intervention) received visits at six and 12 months and routine follow-up by their pediatricians. This intervention was not the primary objective of the present research and participation in the intervention/control group was considered a confounding factor in subsequent statistical analyses.

The study sample consisted of 312 children who completed the initial phase of the study. The anthropometric measures and dietary data for each child were evaluated at three time points $(1,3.9$ and 7.7 years old) at the childs home. The main reason for the loss of subjects was the inability to locate the child's home, usually because the family had moved to another city. Other reasons included refusal to continue in the study and children/maternal death. Race or ethnicity was classified by the interviewer based on skin color. Additional details of the traits studied are described in Vitolo et al. (2008).

The study protocol was approved by the ethics committee of the Universidade Federal de Ciências da Saúde de Porto Alegre and was in accordance with The Code of Eth- ics of the World Medical Association (Declaration of Helsinki) for experiments involving humans. All parents/guardians of the participants provided written informed consent before commencing the study.

\section{Nutritional status and dietary data}

Undergraduate nutrition students were trained to collect data related to the children's anthropometric, dietary, sociodemographic and health status variables. Food intake was assessed through $24 \mathrm{~h}$ dietary recalls. At 1 year old, one $24 \mathrm{~h}$ dietary recall was recorded for each child. At 3.9 and 7.7 years old, two 24 h dietary recalls were recorded on two nonconsecutive days and the mean values were used in the analyses. The first dietary recall was done at the subjects home and the second was done in the municipal health unit. Portion sizes were confirmed with the aid of an album that was specifically designed for this study and contained photographs of utensils and foods, as well as domestic measurements, such as cups, tablespoons and teaspoons. The Nutrition Support Software (Nutwin 1.5, Federal University of São Paulo, São Paulo, Brazil) was used to convert the average sizes into grams for different measurements and to estimate the energy intake from the selected foods (Philippi, 2002; NEPA, 2006).

The energy intake from foods with a high sugar content (sugar-dense foods - SDF) was calculated from the two dietary recalls. The foods were classified as SDF when the sugar content was $\geq 50 \%$ and included items such as soda, Jell-O, candies and artificial juice. The foods were chosen based on the results of a study conducted in Brazil that indicated which foods were most commonly present in childrens diets. The average food intake from the two dietary recalls was used for the analysis.

At 1 year old, each childs height and weight were evaluated. At ages 3.9 and 7.7 years, the height, weight, tricipital and subscapular skinfold thickness and waist circumference were measured. The body mass index (BMI) was calculated as weight $(\mathrm{kg}) /$ height $^{2}\left(\mathrm{~m}^{2}\right)$ and the values were transformed into Z-score (BMIzs). Details of the measurement procedure have been described by Vitolo et al. (2010). The percent variation in BMI for each child was computed with the formula $\left[\left(\mathrm{BMI}_{\mathrm{a}}-\mathrm{BMIb}\right) / \mathrm{BMIb}\right] \mathrm{x} 100$, where $\mathrm{a}=$ final $\mathrm{BMI}$ and $\mathrm{b}=$ initial BMI.

\section{DNA analyses}

At the age of 3.9 years, blood samples were collected for hematological analyses (data not used in this study) and for DNA extraction. The blood samples were collected and transported to the Molecular Biology Laboratory of the Universidade Federal de Ciências da Saúde de Porto Alegre. Genomic DNA was extracted from peripheral blood leukocytes using a standard salting-out procedure (Lahiri and Nurnberger, 1991). The DNA samples are currently stored in this same laboratory. The Ile191 Val polymorphism of the TAS1R2 gene and the $-1266 \mathrm{C} / \mathrm{T}$ polymor- 
phism of the TASIR3 gene were detected by allelic discrimination with TaqMan 5'-nuclease assays using a Step-One-Plus ${ }^{\circledR}$ instrument (real-time PCR, Applied Biosystems, CA, USA). The reactions were run on 96-well plates with fast thermal cycling conditions and the reagent concentrations were: $1 \mathrm{X}$ TaqMan ${ }^{\circledR}$ genotyping master mix, $1 \mathrm{X}$ TaqMan $^{\circledR}$ genotyping assays (C_55646_20 and AHCSYY5), 10 ng of DNA and nuclease-free water.

\section{Statistical analysis}

Allele frequencies were estimated by gene counting. A chi-squared goodness-of-fit test was used to determine whether the distribution of observed genotype frequencies agreed with those expected under Hardy-Weinberg equilibrium. The normality of the variables distribution was analyzed with the Kolmogorov-Smirnov test and by examining the histogram. BMI variation from 3.9 to 7.7 years old, SDF and sugar intake variables were $\ln$ transformed prior to analysis because of their skewed distribution, but the untransformed values are shown. The data for dietary intakes and parameters of adiposity were compared between genotype groups by using a General Linear Model with type III sum of squares. To construct the models, the daily energy intake, SDF and sugar intake, BMI Z-score and BMI variations were included as dependent variables, the genotype groups were used as fixed factors and intervention or control group status, sex and exact age, and exact age difference between each child's evaluations were included as covariates. The models for examining the association of polymorphisms with average daily energy intake, SDF and sugar intake were adjusted for intervention or control group status, sex and exact age. For the BMI Z-scores, only the variable intervention or control group status was included in the model. For variation in BMI, the variables included in the model were the exact age difference between each child's evaluations, intervention or control group status and sex. All tests and transformations were done using the Statistical Package for Social Sciences, version 16.0 (SPSS, Chicago, IL, USA). Values of $\mathrm{p} \leq 0.05$ were considered significant.

\section{Results}

The number of children analyzed differed among the polymorphisms in each phase of the study because some biological samples were not analyzed for all polymorphisms. In samples collected from children at 3.9 years of age $(n=$ 312 ), the average age was $3.98 \pm 1.07$ years old (mean \pm SD), while for children at 7.7 years old $(n=274)$, the average age was $7.71 \pm 0.72$ years. At the age of 7.7 years, $49.3 \%$ of the children were boys and $43.2 \%$ were defined as white.

The allele and genotype frequencies for the TAS1R2 Ile191Val and TAS1R3 -1266 C/T polymorphisms are shown in Table 1. The genotype frequency distributions for
Table 1 - Allele and genotype frequencies for the SNPs TAS1R2 Ile191 Val and TAS1R3 $-1266 C / T$.

\begin{tabular}{lc}
\hline SNP & $\%(\mathrm{n})$ \\
\hline TAS1R2 Ile191Val & \\
Genotypes & \\
Ile/Ile & $51.4(160)$ \\
Ile/Val & $40.6(126)$ \\
Val/Val & $8.0(25)$ \\
Alleles \% & \\
Ile & 72 \\
Val & 28 \\
TAS1R3 -1266 C/T & \\
Genotypes & \\
C/C & $74.3(232)$ \\
C/T & $24.7(77)$ \\
T/T & $1.0(3)$ \\
Alleles \% & \\
C & 87 \\
T & 13 \\
\hline
\end{tabular}

$\mathrm{n}=$ number of carriers of each genotype

the variants studied did not differ from those expected for populations under Hardy-Weinberg equilibrium (TAS1R2 Ile191 Val, $\chi_{\mathrm{GL}=2}^{2}=0.024, \mathrm{p}=0.988$; and TAS1R3 -1266 $\left.C / T, \chi^{2}{ }_{\mathrm{GL}}=2=0.558, \mathrm{p}=0.757\right)$. The most frequent allele for TAS1R2 Ile191Val was *Ile (72\%) and the most frequent allele for TAS1R3 $-1266 C / T$ was ${ }^{*} C(87 \%)$. There were no significant differences in the allele and genotype frequencies between white and non-white children (data not shown).

After adjustment for confounding variables, 3.9 years old children homozygous $(\mathrm{Val} / \mathrm{Val})$ for the TAS1R2 Ile191 Val polymorphism ingested less energy from sugardense foods $(\mathrm{p}=0.027)$ and sugars $(\mathrm{p}=0.025)$ than Ile/Ile and Ile/Val children (Table 2). The percentage variation in BMI from 1 to 3.9 years was not significant among genotypes with the TAS1R2 Ile191 Val polymorphism, but there was a trend towards a smaller reduction in BMI in $\mathrm{Val} / \mathrm{Val}$ homozygotes $(-6.14 \pm 9.13 \%)$ than in *Ile allele carriers $(-9.69 \pm 8.83 \%$ and $-9.82 \pm 8.97 \% ; \mathrm{p}=0.052)$ in this period (Table 2). For 1 and 7.7 years old children, no association was detected between food intake and nutritional status for this variant, but at 7.7 years there was a tendency for $\mathrm{Val} / \mathrm{Val}$ homozygotes to have a greater BMI z-score; there was also greater variation in BMI in 3.9 to 7.7 years old children than in the corresponding *Ile allele carriers. The polymorphism $T A S 1 R 3-1266 C / T$ was not associated with food intake and nutritional status at the three evaluations done in this study.

\section{Discussion}

This study examined the association between two genetic variants of T1R taste receptors and food intake and anthropometric parameters during childhood. The Ile191 Val polymorphism of the TASIR2 gene was found to 


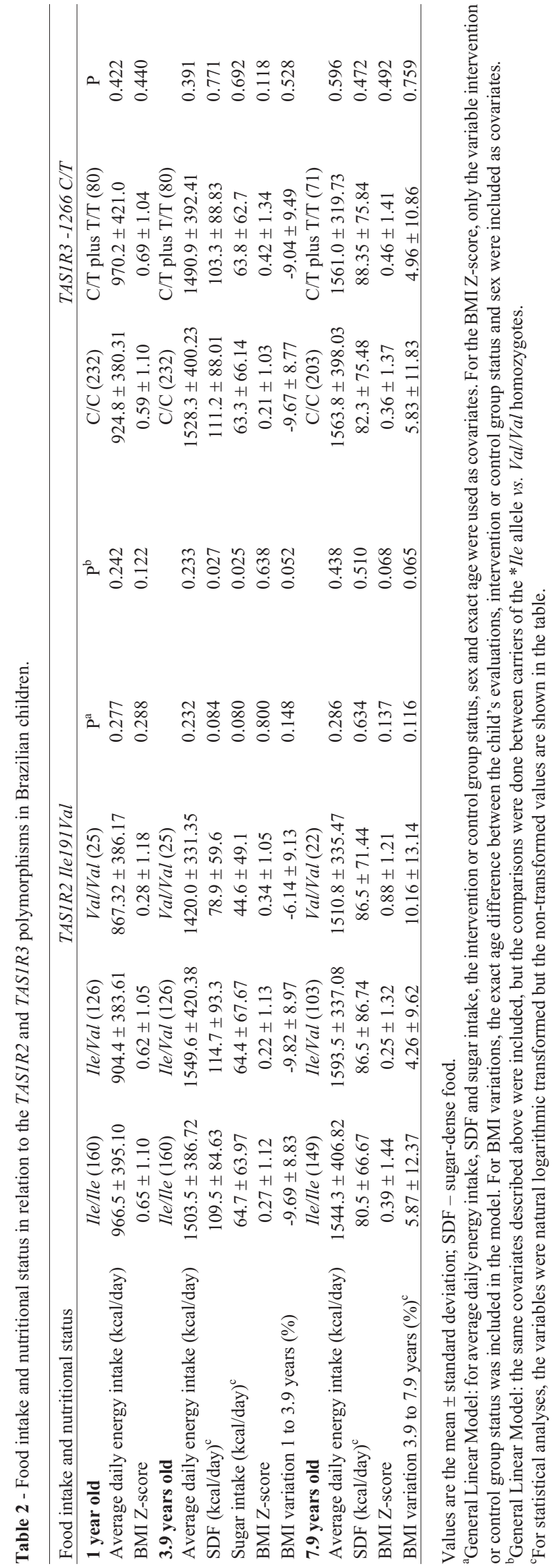

be associated with differences in the habitual consumption of SDF and sugar intake in children at the age of 3.9 years. The decrease in SDF and sugar intake in $\mathrm{Val} / \mathrm{Val}$ homozygotes was not significantly associated with anthropometric parameters or with variation in weight gain in this cohort. Additionally, contrary to our initial hypothesis, there was a trend towards a higher BMI z-score at 7.7 years old and greater weight gain at 3.9 and 7.7 years old in $\mathrm{Val} / \mathrm{Val}$ homozygotes when compared with *Ile allele carriers. The lack of association of this polymorphism with anthropometric parameters and the trend detected in the opposite direction may be explained by the observation that greater consumption of sugar and SDF did not increase the mean total daily energy intake in $\mathrm{Val} / \mathrm{Val}$ homozygous children.

The functional significance of the Ile191 Val polymorphism is not yet known and there is currently no information on this matter in the PolyPhen database (http://genetics.bwh.harvard.edu/pph/data/). This variation is located in the conserved large extracellular domain that is one of the ligand-binding regions of the sweet taste receptor (Liao and Schultz, 2003; Nie et al., 2005; Kim et al., 2006). Eny et al. (2010) hypothesized that the Ile191 Val variant may not be a causal polymorphism but may instead be in linkage disequilibrium with a causal polymorphism. These authors studied the association between this polymorphism and food intake in young adults and diabetics and noted a lack of association with food intake in lean individuals; however, as also observed in the present study, in overweight individuals (BMI $>25)$ there was a decrease in total carbohydrates, available carbohydrates and total sugar intake for carriers of the *Val allele compared with Ile/Ile homozygous individuals.

In the present study, $-1266 C / T$ polymorphism of the $T A S 1 R 3$ gene was not associated with food intake and nutritional status in three evaluations of the same children in early childhood. The TASIR3 gene encodes the receptor subunit of the sweet and the umami receptors. The T1R2T1R3 taste receptor responds to sweet-tasting compounds, such as sugars, high-potency sweeteners and some Damino acids, whereas T1R1-T1R3 heteromers comprise an umami taste receptor that is sensitive to L-amino acids (Nelson et al., 2001; Zhao et al., 2003; Nie et al., 2005). Kim et al. (2006) proposed that human populations likely show little variation with respect to umami perception, which is controlled by one major form of the receptor that is optimized for detecting glutamate, while there may be a higher level of variation with respect to sweet perception (Kim et al., 2006). Studies associating TAS1R3 gene polymorphisms with variations in sensitivity to sweet taste in humans are still limited. Fushan et al. (2009) evaluated 144 unrelated individuals and observed a $25 \%$ reduction in the sensitivity to sweet taste in $\mathrm{C} / \mathrm{T}$ heterozygous individuals. The results of the latter study are not comparable with ours because we evaluated food intake and nutritional status in children and not the sensitivity to sweetness. We hypothe- 
size that to detect an association between a genetic variant of these taste receptors and the habitual consumption of sugar, this variation needs to have a strong effect on protein functionality or expression. In this context, the approach of Fushan et al. (2009) is more sensitive for detecting small genetic effect.

Our findings suggest that genetic variation in one component of the T1R2-T1R3 heterodimer may account for interindividual differences in the consumption of sweet foods, although this association was not reflected in modifications in total energy intake or in anthropometric parameters. Our findings contribute to knowledge in the area of taste genetics and may help to explain the different ability of individuals to alter their dietary intake in response to dietary advice. However, this study has some limitations. Only one polymorphism was evaluated for each gene and these polymorphisms were selected based on previous studies describing their possible association with food intake and sweet sensibility. Another relevant feature was the age of the children. In view of their young age, the children may not have had the freedom to choose their foods and they may instead have been supplied with foods that were palatable to adults. In addition, the participants may have consumed all the available foods equally, regardless of whether they presented a greater sensitivity or not, and our data on food intake do not necessarily reflect the sweet sensitivity and food preferences. Furthermore, the food categories analyzed (SDF and sugar intake) may not accurately reflect the issue of palatability and sweet taste because some foods, such as soda, may have a high concentration of sugar but be perceived as less sweet. However, the association detected between genetic variants of the sweet taste receptor gene and SDF and sugar intake makes biological sense. To the best of our knowledge, no other studies have evaluated the association between genetic variations in the taste receptor genes and food intake and nutritional status at different stages of childhood. The results described here may open new perspectives for researchers and contribute to the study of childhood dietary patterns and nutritional status.

\section{Acknowledgments}

This work was supported by two Brazilian federal government agencies, the National Council of Technological and Scientific Development (CNPq) and the Support Program for Centers of Excellence (PRONEX), as well as the Rio Grande do Sul State Research Foundation (FAPERGS). All authors reviewed the manuscript and approved the final version.

\section{References}

Allison DB, Downey M, Atkinson RL, Billington CJ, Bray GA, Eckel RH, Finkelstein EA, Jensen MD and Tremblay A (2008) Obesity as a disease: A white paper on evidence and arguments commissioned by the council of The Obesity Society. Obesity 16:1161-1177.

Brasil Ministério da Saúde and Organização Pan-Americana da Saúde (2002) Dez Passos da Alimentação Saudável: Guia Alimentar para Crianças Menores de Dois Anos. Ministério da Saúde, Brasília, 50 p.

Eny KM, Wolever TM, Corey PN and Sohemy AE (2010) Genetic variation in TAS1R2 (Ile191Val) is associated with consumption of sugars in overweight and obese individuals in 2 distinct populations. Am J Clin Nutr 92:1501-1510.

Fushan AA, Simons CT, Slack JP, Manichaikul A and Drayna D (2009) Allelic polymorphism within the TAS1R3 promoter is associated with human taste sensitivity to sucrose. Curr Biol 19:1288-1293.

Garcia-Bailo B, Toguri C, Eny KM and El-Sohemy A (2009) Genetic variation in taste and its influence on food selection. $\mathrm{J}$ Integr Biol 13:69-80.

Hoon MA, Adler E, Lindemeier J, Battey JF, Ryba NJP and Zuker CS (1999) Putative mammalian taste receptors: A class of taste-specific GPCRs with distinct topographic selectivity. Cell 96:541-551.

Kim UK, Wooding S, Riaz N, Jorde LB and Drayna D (2006) Variation in the human TAS1R taste receptors genes. Chem Senses 31:599-611.

Lahiri DK and Nurnberger JI (1991) A rapid non-enzymatic method for the preparation of 413 HMW DNA from blood for RFLP studies. Nucleic Acids Res 19:5444.

Leterne A, Brun L, Dittmar A and Robin O (2008) Autonomic nervous system responses to sweet taste: Evidence for habituation rather than pleasure. Physiol Behav 93:994-999.

Liao J and Schultz PG (2003) Three sweet receptors genes are clustered in human chromosome. Mamm Genome 14:291-301.

Nelson G, Hoom MA, Chandrashekar J, Zhang Y, Ryba NJP and Zuker CS (2001) Mammalian sweet taste receptors. Cell 106:381-390.

NEPA (2006) Tabela Brasileira de Composição de Alimentos (TACO): Versão 2. Núcleo de Estudos e Pesquisas em Alimentação NEPA-UNICAMP, Campinas, 114 p.

Nie Y, Vigues S, Hobbs JR, Conn GL and Munger SD (2005) Distinct contributions of T1R2 e T1R3 taste receptor subunits to the detection of sweet stimuli. Curr Biol 15:1948-1952.

Oliveira CL and Fisberg M (2003) Obesidade na infância e adolescência - Uma verdadeira epidemia. Arq Bras Endocrinol Metab 47:107-108.

Philippi ST (2002) Tabela de Composição de Alimentos: Suporte para Decisão Nutricional. 2 ed. Editora Gráfica Coronário, Brasília, 135 p.

Vitolo MR, Bortolini GA, Dal Bo Campagnolo P and Feldens CA (2008) Effectiveness of a nutrition program in reducing symptoms of respiratory morbidity in children: A randomized field trial. Prev Med 47:384-388.

Vitolo MR, Rauber F, Campagnolo PD, Feldens CA and Hoffman DJ (2010) Maternal dietary counseling in the first year of life is associated with a higher healthy eating index in childhood. J Nutr 140:2002-2007.

Zhao GQ, Zhang Y, Hoom MA, Chandrashekar J, Erlenbach I, Ryba NJ and Zuker CS (2003) The receptors of mammalian sweet and umami taste. Cell 115:255-266. 


\section{Internet Resources}

PolyPhen Data base, http://genetics.bwh.harvard.edu/pph/data/ (June, 2016).

WHO (2010) World Health Organization. Regional office for Europe, http://www.euro.who.int/en/what-we-do/health-topics/diseases-and-conditions/obesity (June, 2016).

Associate Editor: Jorge Lopez-Camelo

License information: This is an open-access article distributed under the terms of the Creative Commons Attribution License (type CC-BY), which permits unrestricted use, distribution and reproduction in any medium, provided the original article is properly cited. 